\title{
High-Fat Diet Increases HMGB1 Expression and Promotes Lung Inflammation in Mice Subjected to Mechanical Ventilation
}

\author{
Ana Beatriz Farias de Souza, ${ }^{1}$ Máira Tereza Talma Chírico, ${ }^{1}$ Christiane Teixeira Cartelle, ${ }^{2}$ \\ Guilherme de Paula Costa, ${ }^{1}$ André Talvani ${ }^{D},{ }^{1}$ Sílvia Dantas Cangussú, ${ }^{1}$ \\ Rodrigo Cunha Alvim de Menezes, ${ }^{1}$ and Frank Silva Bezerra ${ }^{1}{ }^{1}$ \\ ${ }^{1}$ Department of Biological Sciences (DECBI), Center of Research in Biological Sciences (NUPEB), Federal University of Ouro Preto \\ (UFOP), Ouro Preto, MG, Brazil \\ ${ }^{2}$ Department of Pathology, Institute of Biological Sciences, Federal University of Minas Gerais, Belo Horizonte, MG, Brazil \\ Correspondence should be addressed to Frank Silva Bezerra; franksbezerra@hotmail.com
}

Received 26 October 2017; Revised 16 December 2017; Accepted 21 December 2017; Published 12 February 2018

Academic Editor: Francisco J. Romero

Copyright (c) 2018 Ana Beatriz Farias de Souza et al. This is an open access article distributed under the Creative Commons Attribution License, which permits unrestricted use, distribution, and reproduction in any medium, provided the original work is properly cited.

\begin{abstract}
This study aims to evaluate the effects of a high-fat diet and mechanical ventilation on the pulmonary and systemic inflammatory response in C57BL/6 mice. Male C57BL/6 mice were divided into two groups: one received a standard diet, and the other received a high-fat diet. After 10 weeks, the groups were further divided into two groups each: control group (CG), mechanical ventilation group (MVG), diet group (DG), and diet mechanical ventilation group (DMVG). MVG and DMVG underwent mechanical ventilation for 60 minutes. All animals were euthanized for subsequent analysis. Animals receiving a high-fat diet presented higher body mass, adipose index, and greater adipocyte area. In the lung, the expression of HMGB1 was greater in DG and DMVG than in CG and MVG. CCL2 and IL-22 levels in MVG and DMVG were increased compared to those in CG and DG, whereas IL-10 and IL-17 were decreased. Superoxide dismutase activity was higher in MVG and DMVG than in CG. Catalase activity was lower in DG than in CG, and in MV groups, it was lower than that in CG and DG. MV and obesity promote inflammation and pulmonary oxidative stress in adult C57BL/6 mice.
\end{abstract}

\section{Introduction}

The prevalence of obesity is increasing globally at alarming rates, and according to the World Health Organization, the worldwide prevalence of obesity has more than doubled since 1980 and is associated with several comorbidities [1, 2]. Although its pathogenesis is not completely understood, the development of obesity does not involve a single cause but a complex combination of several conditions caused by multiple factors resulting in the disease phenotype. The etiology of obesity involves genetic factors, which can be attenuated or exacerbated by dietary, environmental, and psychosocial factors [3]. Obesity, particularly the expansion of visceral adipose tissue, promotes increased production of adhesion molecules, recruitment and differentiation of monocytes, and, consequently, elevated production of cytokines and adipokines [1, 4]. Moreover, adipose tissue accumulation in the thoracic cavity and abdomen alters respiratory physiology causing an increased load on the respiratory musculature, leading to decreased chest wall compliance and increased resistance, altered ventilation perfusion relation, lung volume, and capacity [5-7]. Recent data indicate that in intensive care units, approximately $20 \%$ of patients are obese or severely obese [8]. These patients have more complications and need longer hospitalization and mechanical ventilation [5].

Mechanical ventilation (MV) is a tool used for patients with respiratory insufficiency. Although it presents an important therapeutic role, MV can cause lung injury or aggravate preexisting injury, resulting in ventilator-induced lung injury (VILI) [9]. The mechanisms by which VILI develops are not fully described, but studies demonstrate that 
cyclic stretching caused by MV may damage alveolar epithelial cells and increase permeability, which is associated with leukocyte recruitment into the air space, resulting in the production of inflammatory cytokines and reactive oxygen species (ROS) [10]. ROS production at high concentrations is related to redox imbalance, characterized by an altered ratio of oxidants to antioxidants, where the concentration of reactive species transiently or chronically increases the damage in the regulation of cellular metabolism, thus injuring the cellular constituents $[11,12]$. Despite the clinical and epidemiological relevance, few studies have explored the association between obesity and MV and its deleterious effects on the body. In this study, we evaluated the effects of a highfat diet and mechanical ventilation on redox imbalance and on the pulmonary and systemic inflammatory response in C57BL/6 mice.

\section{Methods}

2.1. Animals. Forty male C57BL/6 mice, aged 7 to 8 weeks, were obtained from the Animal Science Center (CCA) of the Federal University of Ouro Preto (UFOP). The animals were kept under controlled conditions of temperature $\left(21 \pm 2^{\circ} \mathrm{C}\right)$, humidity $(50 \pm 10 \%)$, and luminosity (12 hours light/dark cycle). The experimental procedures were performed in accordance with the Ethical Principles of Animal Experimentation established by the Ethics Committee on Animal Use (CEUA) of UFOP and approved by protocol number 2015/41.

2.2. Experimental Design and Diet Composition. First, the animals were divided into two groups $(n=20)$ according to the type of diet provided: control group and diet group. The control group received standard chow (Nuvilab ${ }^{\circledR}$ ) containing 3.5\% lipids; the diet group was provided a high-fat diet with $45 \%$ lipids (based on formula D12451, Research Diets Inc., New Brunswick, NJ, USA) [13]. The animals were evaluated weekly for weight gain on a digital balance (Marte Scientific and Industrial Instrumentation ${ }^{\circledR}$, São Paulo, BR). At the end of 10 weeks of this period, the animals from each group were randomly divided into two groups of 10 animals each: control group (CG), mechanical ventilation group (MVG), diet group (DG), and diet mechanical ventilation group (DMVG). Animals from the CG and DG were maintained on spontaneous ventilation, and MVG and DMVG were subjected to mechanical ventilation for 60 minutes.

2.3. Mechanical Ventilation. Twenty-four hours after the end of the nutritional protocol, the animals of the MVG and DMVG were sedated and anesthetized by intraperitoneal administration of ketamine $(10 \mathrm{mg} / \mathrm{kg})$ and xylazine $(8 \mathrm{mg} / \mathrm{kg})$ and placed on a surgical table for a median incision to be performed in the anterior cervical region. The musculature was dissected with the aid of a hemostatic forceps, the trachea was exposed, and an incision was made with a catheter to connect the animal to the MiniVent ventilator (Harvard Apparatus, Massachusetts, USA). The animals were ventilated in the volume-controlled mode according to the following parameters: tidal volume of $7 \mathrm{~mL} / \mathrm{kg}$, respiratory rate of 150 breaths/minute, and inspired fraction of oxygen at $21 \%$. All animals subjected to mechanical ventilation were paralyzed using suxamethonium chloride $(0.3 \mathrm{mg} / \mathrm{kg}$, intravenous). Peripheral saturation and control of body temperature were assessed throughout the period for which the animals were ventilated.

2.4. Euthanasia. After the mechanical ventilation in the experimental protocol, blood was collected by cardiac puncture and placed in polypropylene tubes containing $15 \mu \mathrm{L}$ of anticoagulant for the evaluation of hematological parameters using an electronic counting device (Mindray ${ }^{\circledR}$ Bio-Medical Electronics Co. Ltd., Shenzhen, China) [14].

2.5. Collection and Analysis of Bronchoalveolar Lavage Fluid (BALF). Immediately after euthanasia, the thorax of each animal was opened, the left main bronchus was clamped, the trachea was cannulated, and the left lung was washed with $1.5 \mathrm{~mL}$ of saline solution $(3 \times 500 \mu \mathrm{L})$. The samples were kept on ice until the end of the procedure to avoid cell lysis. A Neubauer chamber was used for the total leukocyte count of BALF. To determine the differential cell count, $250 \mu \mathrm{L}$ samples were centrifuged in a cytocentrifuge (INBRAS health equipment, São Paulo, BR) and stained with a fast panoptic coloration kit (Laborclin, Paraná, BR), and 100 cells per slide were counted; each procedure was performed by two evaluators [15].

2.6. Tissue Processing and Homogenization. After BALF collection, the right ventricle was perfused with saline solution to remove blood from the lungs. The right lung was clamped, and the left lung instilled with $4 \%$ buffered formalin ( $\mathrm{pH} 7.2$ ) at a pressure of $25 \mathrm{cmH}_{2} \mathrm{O}$ for 2 minutes, via the trachea. The left lung was then removed and immersed in fixative solution for 48 hours. The samples were processed, and slides were stained with hematoxylin and eosin ( $\mathrm{H} \& \mathrm{E})$ to perform stereological analyses or used for immunohistochemistry. The right lung was homogenized with $1.5 \mathrm{~mL}$ of phosphate buffer ( $\mathrm{pH} 7.4$ ); the samples were centrifuged for 10 minutes at $10,000 \mathrm{rpm}$; the supernatant was collected and stored at $-80^{\circ} \mathrm{C}$ for biochemical analysis [14].

2.7. Calculation of Body Fat Index. The adipose tissue (mesenteric, retroperitoneal, and epididymal) was removed and weighed to determine the body fat index, which was calculated according to the equation described by Catta-Preta et al. [16].

2.8. Morphometric Analyses of Lung and Adipose Tissue. Morphometric analyzes were performed on lungs and epididymal adipose tissue stained with H\&E. Twenty random fields of lung histological slides were photographed using a light microscope equipped with a Leica BM5000 digital camera (Leica DFC 300 FX) coupled to the Leica Application Suite image capture software using a 40x microscopic objective. The volume density analysis of the alveolar septum ( Vv) was performed in a test system composed of 16 points and a known test area, as described by Mandarim-de-Lacerda [17] and Campos et al. [15]. 
For analyzing the adipocyte area, the histological sections were photographed using the same microscope with a $10 \mathrm{x}$ microscopic objective. Ten random fields were analyzed, and the mean area of the adipocytes was obtained by analyzing 50 adipocytes per slide in ImageJ 1.6.0 software (Wayne Rasband-National Institutes of Health, USA) [14, 18].

2.9. Immunohistochemistry. Two histological sections of each animal were stained with HMGB1 (EPR3507) (Abcam, UK) by immunohistochemistry. The slides were deparaffinized in xylene and rehydrated in decreasing ethanol concentrations. Subsequently, antigen recovery was performed with EDTA solution pH 9.0 in a water bath at $96^{\circ} \mathrm{C}$ for 20 minutes. Endogenous peroxidase activity was blocked with $\mathrm{H}_{2} \mathrm{O}_{2}$ solution in methanol, twice for 15 minutes. Nonspecific binding was blocked with $3 \%$ skim milk (MOLICO, Nestlé Brasil Ltda., Araçatuba, SP) in phosphate buffered saline (PBS), followed by blocking with $2 \%$ BSA (bovine serum albumin; Inlab, Brazil) in PBS and blocking with normal goat serum (NGS) diluted in PBS at $1: 20$. The slides were incubated for 30 minutes in each blocking solution and washed in PBS after each incubation period, except for the blocking performed with NGS. The slides were incubated overnight at $4^{\circ} \mathrm{C}$ with rabbit primary monoclonal anti-HMGB1 diluted at $1: 250$. After incubation with the primary antibody, the slides were washed three times with PBS, followed by incubation with the biotinylated secondary antibody in a humid chamber at $37^{\circ} \mathrm{C}$ for 30 minutes. Finally, the slides were incubated under the same conditions with the streptavidin/peroxidase complex (Dako, Santa Clara, CA, USA). Peroxidase activity was detected using the 3,3-diaminobenzidine substrate (DAB). All slides were stained with Harris hematoxylin. For each histological section stained with HMGB1, a control was prepared in which the primary antibody was suppressed.

Morphometric analysis of the sections stained by immunohistochemistry was performed in 20 random fields of the slides photographed at a magnification of 20x using ImageJ 1.6.0 software (Wayne Rasband-National Institutes of Health, USA). In each field, the total number of nuclei and the number of nuclei labeled for the antibody used were counted, and the ratio of labeled nuclei/total nuclei was calculated [19].

2.10. Analysis of Antioxidant Defense and Biomarkers of Oxidative Stress. Superoxide dismutase activity was measured in the tissue homogenate according to the method described by Marklund and Marklund [20], which is based on the ability of SOD to inhibit pyrogallol autoxidation. Catalase activity was measured according to the method described by Aebi [21] from the decreased $\mathrm{H}_{2} \mathrm{O}_{2}$ at an absorbance of $240 \mathrm{~nm}$. Glutathione dosage was adapted from a Sigma commercial kit (CS0260; Sigma, St. Louis, MO, USA), which uses a kinetic method to measure the total glutathione levels (GSH + GSSG) in biological samples by reducing 5,5' -dithio-bis-(2-nitrobenzoic acid) to 5-thio-2-nitrobenzoic acid [22]. Formation of thiobarbituric acid reactive substances (TBARS) was used to measure lipid peroxidation; the method is based on the ability of thiobarbituric acid to bind to oxidized lipids as previously described by Buege and Aust [23]. For determination of carbonylated proteins, a protocol adapted from the method described by Reznick and Packer [24] was used. The total protein content in the samples was determined by the Bradford method [25].

2.11. Immunoenzymatic Assay for Inflammatory Markers. The pulmonary homogenate was used for analyses of monocyte chemoattractant protein-1 (MCP-1 or CCL2), regulated on activation, normal $\mathrm{T}$ cell expressed and secreted (RANTES or CCL5), and interleukins 10,17 , and 22 . The assays were performed in 96-well plates; $100 \mu \mathrm{L}$ of monoclonal antibody was added to the protein (or peptide), which was diluted in PBS containing $0.1 \%$ bovine serum albumin (BSA; SigmaAldrich, Billerica, MA, USA). After incubation for 12 hours at $37^{\circ} \mathrm{C}$, the plates were blocked with $300 \mu \mathrm{L} /$ well of a PBS solution containing $1 \% \mathrm{BSA}$ for 1 hour at $37^{\circ} \mathrm{C}$. The samples were applied in a volume of $100 \mu \mathrm{L}$ to each well. The staining intensity was measured using an enzyme-linked immunosorbent assay (ELISA) reader at a wavelength of $490 \mathrm{~nm}$. All ELISA kits were purchased from PeproTech (Ribeirão Preto, Brazil).

2.12. Statistical Analysis. Parametric distribution of the data was evaluated using the Kolmogorov-Smirnov normality test. Parametric data were expressed as mean and standard error of the mean; nonparametric data were expressed as median, minimum, and maximum values. Analysis of the body mass data was performed by two-way ANOVA by the Bonferroni posttest. For comparison of two or more groups, a one-way ANOVA followed by Tukey's posttest was used for comparing two or more groups. For nonparametric data, the Kruskal-Wallis test was applied with Dunn's posttest. Significant differences were considered at $p<0.05$. All statistical analyses were performed using GraphPad Prism software 5.0 (San Diego, CA, USA).

\section{Results}

3.1. Effects of High-Fat-Diet Administration. Since the third week of the experiment, the diet group presented higher body mass than did the group that received a standard diet. The difference between the groups was maintained until the end of the experiment (Figure 1(a)). The animals in DG and DMVG presented higher adiposity index and adipocyte area than did those in to CG and MVG $(p<0.0001)$ (Figures 1(b)-1(d)).

3.2. Total and Differential Leukocyte Count in Blood. In peripheral blood, the animals in MVG and DMVG presented a higher leukocyte count than did those in CG and DG $(p<0.0001, F=11.5)$. The neutrophil count was higher in MVG and DMVG than in CG and DG; in MVG, the count was higher than that in DMVG $(p<0.0001, F=181.2)$. The monocyte count was higher in groups subjected to MV than that in CG and DG $(p<0.0001, F=15.0)$ (Table 1).

3.3. Cell Recruitment to Bronchoalveolar Lavage Fluid. Highfat diet, mechanical ventilation, and the combination of diet and ventilation caused a higher recruitment of cells to the lung when compared to that in the control group $(p<0.0001$, 


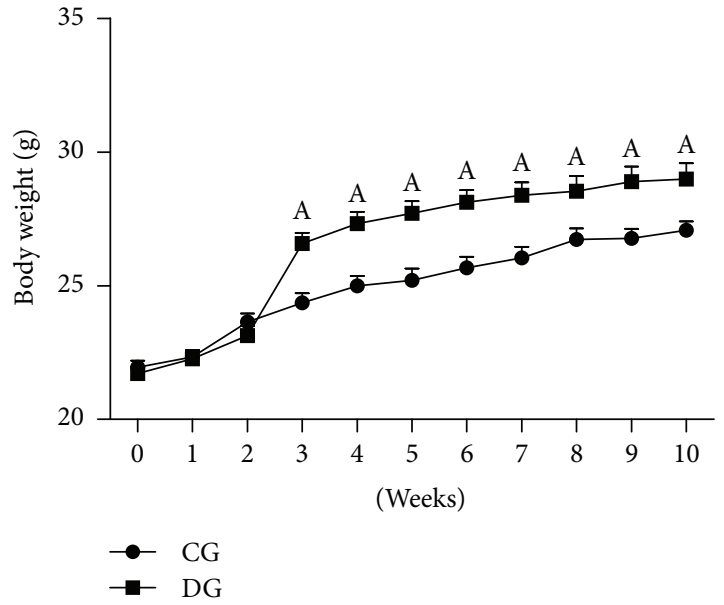

(a)

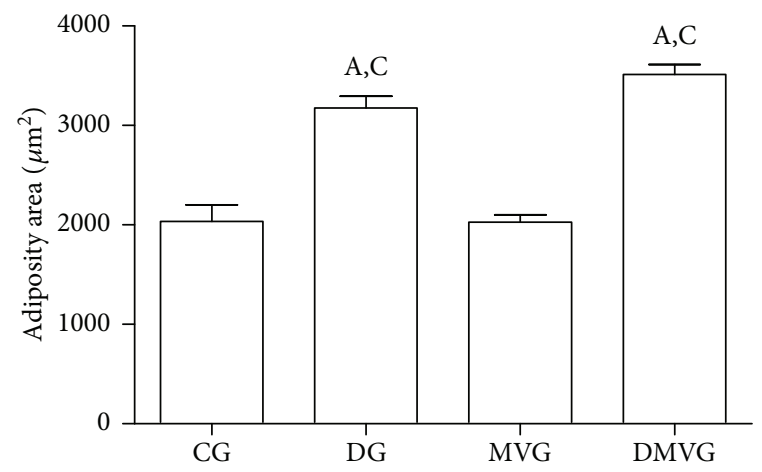

(c)

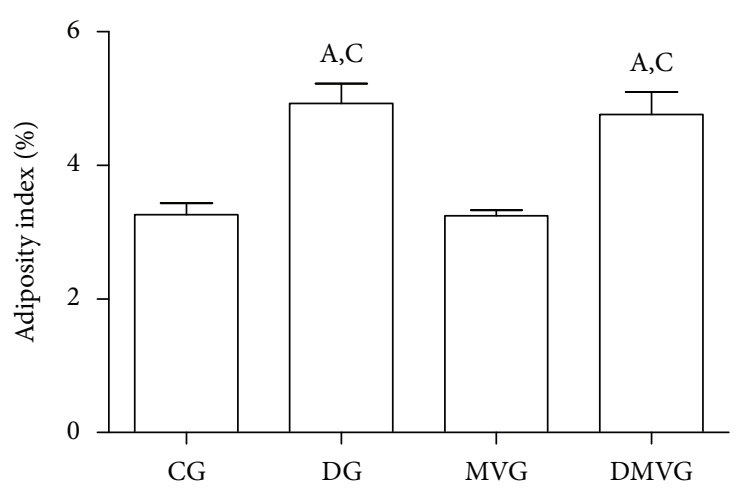

(b)

CG

DG

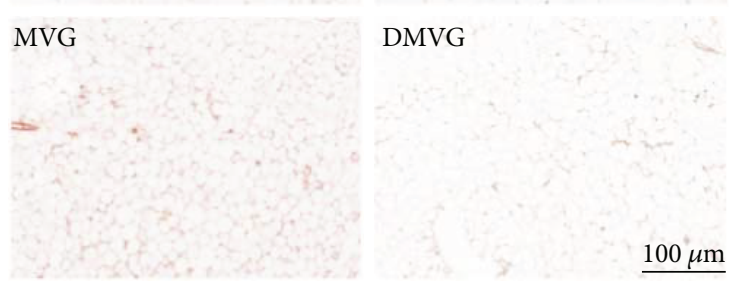

(d)

FIGURE 1: Effects of the hyperlipidic diet on body mass and adipose tissue. (a) Body mass gain over the 10-week experiment. (b) Body adiposity index. (c) Area of adipocytes. (d) Histological section of epididymal adipose tissue stained with hematoxylin and eosin. Bar $=100 \mu \mathrm{m}$. For (a), data are expressed as mean \pm standard error of the mean $(n=20)$. (a) represents difference compared to the control group $p<0.05$ using two-way ANOVA followed by the Bonferroni posttest. For (b) and (c), (A) and (C) represent a significant difference in relation to CG and MVG. Data are expressed as mean \pm standard error of the mean $(n=10)$. Analysis was performed by one-way ANOVA followed by Tukey's posttest $(p<0.05)$.

TABLE 1: Total and differential evaluation of blood cells from experimental groups.

\begin{tabular}{lcccc}
\hline & CG & DG & MVG & DMVG \\
\hline Leukocytes $\left(\times 10^{3} / \mathrm{mL}\right)$ & $2.61 \pm 0.37$ & $2.14 \pm 0.22$ & $4.72 \pm 0.35^{\mathrm{a}, \mathrm{b}}$ & $3.90 \pm 0.36^{\mathrm{a}, \mathrm{b}}$ \\
Lymphocytes $\left(\times 10^{3} / \mathrm{mL}\right)$ & $2.36 \pm 0.29$ & $1.76 \pm 0.20$ & $2.15 \pm 0.22$ & $2.02 \pm 0.20$ \\
Neutrophils $\left(\times 10^{3} / \mathrm{mL}\right)$ & $0.06 \pm 0.01$ & $0.06 \pm 0.01$ & $0.92 \pm 0.01^{\mathrm{a}, \mathrm{b}, \mathrm{d}}$ & $0.38 \pm 0.05^{\mathrm{a}, \mathrm{b}}$ \\
Monocytes $\left(\times 10^{3} / \mathrm{mL}\right)$ & $0.19 \pm 0.02$ & $0.32 \pm 0.04$ & $1.65 \pm 0.29^{\mathrm{a}, \mathrm{b}}$ & $1.50 \pm 0.20^{\mathrm{a}, \mathrm{b}}$ \\
\hline
\end{tabular}

(a) represents significant difference between groups when compared to CG. (b) represents significant difference between groups when compared to DG. (d) represents significant difference between groups when compared to DMVG. Data are expressed as mean \pm standard error of the mean $(n=10)$ and were analyzed by one-way ANOVA followed by Tukey's posttest $(p<0.05)$. CG: control group; DG: diet group; MVG: mechanical ventilation group; DMVG: diet mechanical ventilation group.

$F=109.8)$. The animals in DG had a higher macrophage population in BALF than had those in CG $(p<0.0001$, $F=76.8$ ). The number of macrophages, neutrophils, and lymphocytes in MVG and DMVG was increased in comparison to that in CG and DG $(p<0.0001, F=24.9)$ (Table 2).
3.4. Evaluation of Cytokine and Chemokine Levels in the Pulmonary Parenchyma. The inflammatory markers CCL2, CCL5, IL-17, IL-22, and IL-10 were analyzed in the lung parenchyma to assess the inflammatory state of the lungs. The levels of CCL2 $(p<0.0001, F=13.1)$ and 
TABLE 2: Effects of diet and mechanical ventilation on cell recruitment to BALF.

\begin{tabular}{lcccc}
\hline & CG & DG & MVG & DMVG \\
\hline Leukocytes $\left(\times 10^{3} / \mathrm{mL}\right)$ & $83.33 \pm 4.41$ & $119.00 \pm 5.04^{\mathrm{a}}$ & $204.4 \pm 6.47^{\mathrm{a}, \mathrm{b}}$ & $228.0 \pm 9.31^{\mathrm{a}, \mathrm{b}, \mathrm{c}}$ \\
Macrophages $\left(\times 10^{3} / \mathrm{mL}\right)$ & $78.46 \pm 5.30$ & $108.40 \pm 4.74^{\mathrm{a}}$ & $178.30 \pm 7.03^{\mathrm{a}, \mathrm{b}}$ & $199.97 \pm 8.80^{\mathrm{a}, \mathrm{b}}$ \\
Neutrophils $\left(\times 10^{3} / \mathrm{mL}\right)$ & $1.61 \pm 0.35$ & $1.92 \pm 0.37$ & $8.00 \pm 1.52^{\mathrm{a}, \mathrm{b}}$ & $8.13 \pm 1.10^{\mathrm{a}, \mathrm{b}}$ \\
Lymphocytes $\left(\times 10^{3} / \mathrm{mL}\right)$ & $3.26 \pm 0.52$ & $8.68 \pm 1.01$ & $18.10 \pm 2.30^{\mathrm{a}, \mathrm{b}}$ & $19.90 \pm 2.45^{\mathrm{a}, \mathrm{b}}$ \\
\hline
\end{tabular}

(a) represents significant difference between groups when compared to CG. (b) represents significant difference between groups when compared to DG. (c) represents significant difference between groups when compared to MVG. Data are expressed as mean \pm standard error of the mean $(n=10)$ and were analyzed by one-way ANOVA followed by Tukey's posttest $(p<0.05)$. CG: control group; DG: diet group; MVG: mechanical ventilation group; DMVG: diet mechanical ventilation group.

IL-22 $(p<0.0001, F=14.6)$ were higher in the groups subjected to MV compared to those in the animals on spontaneous ventilation whereas the levels of IL-17 $(p<0.0001$, $F=11.6)$ and IL-10 $(p<0.0001, F=15.4)$ were lower in these groups (Table 3 ).

\subsection{Effects of Mechanical Ventilation and Obesity on Oxidative} Stress Biomarkers. Lipid peroxidation in MVG was higher than that in the other experimental groups $(p=0.0004$, $F=7.9)$. The protein oxidation levels were higher in MVG and DMVG than those in CG and DG $(p<0.0001, F=18.6)$. Regarding the activity of antioxidant enzymes, SOD activity was higher in MVG and DMVG than in CG $(p=0.0067$, $F=5.0)$. CAT activity was lower in DG than in CG, and in groups subjected to MV, enzyme activity was even lower in comparison with that in CG and DG $(p<0.0001, F=$ 117.1). The GSH/GSSG ratio was lower in MVG than in CG $(p<0.03, F=3.4)$ (Table 4$)$.

3.6. Analysis of HMGB1 in Lung Parenchyma. The morphometric analysis of HMGB1 immunohistochemistry showed a higher number of nuclei labeled with the antibody in DG and DMVG than in CG and MVG, which can be observed by the highest ratio of marked nuclei/total nuclei (Figure 2).

3.7. Morphometric Evaluation of the Pulmonary Parenchyma. The stereological analysis showed no differences in volume density of alveolar air ( $\mathrm{Vv}[\mathrm{a}])$ and in volume density of alveolar septa (Vv[sa]) (Figure 3).

\section{Discussion}

In this study, we evaluated the effects of a high-fat diet and mechanical ventilation on the inflammatory response and redox imbalance. The effects of diet were observed under body mass, adiposity index and adipocyte area, influx of cells to the lung parenchyma, hematological parameters, oxidative stress analysis, and inflammatory markers in the lungs.

Studies have shown that the composition of the diet offered in experimental models influences the development of obesity and the diseases associated with obesity, since nutrients act as cellular signals [26, 27]. In our study, animals that received the high-fat diet presented higher body mass, increased body adiposity, and greater area of adipocytes than did the control animals. This diet has been previously used in experimental models for the induction of obesity and is accompanied by an increase in body mass [16, 28]. This increase is directly related to the increase in adiposity and the greater area of adipocytes, since the excessive caloric intake is associated with storage of excess energy in adipose tissue in the form of lipids, leading to its expansion $[16,29]$.

Obesity is characterized by high body mass and also by systemic and local inflammatory changes, with an increase in the production of proinflammatory cytokines [1]. In this context, the role of HMGB1 protein in tissue inflammation has been studied. In our study, we observed that animals receiving a high-fat diet showed a higher number of nuclei labeled for the HMGB1 protein. There are no published studies demonstrating the expression of this protein in lungs in an experimental model of obesity, but some previous work has shown that obesity is associated with increased expression of HMGB1 and inflammatory cytokines in the adipose tissue $[30,31]$. We believe that our finding is related to the chronic and systemic inflammatory state caused by obesity and may be related to the still-unknown role of HMGB1 in disease development [31].

Both obesity and mechanical ventilation have been shown to generate inflammatory processes $[14,32]$. In order to evaluate whether mechanical ventilation and a diet rich in saturated lipids caused an inflammatory response, the leukocytes in blood and BALF were examined. Our results demonstrate that both insults generated an inflammatory response. Animals that received the high-fat diet presented higher recruitment of macrophages to the pulmonary parenchyma, which can be explained by the fact that these cells are fundamental regulators of immune responses and inflammation in obesity [33]; our results corroborate the findings of Tashiro et al. [34] who observed an increased number of macrophages in the bronchoalveolar lavage of mice provided a high-fat diet. In groups subjected to MV, there was an increase in the macrophages, neutrophils, and lymphocytes in BALF, and the monocytes and neutrophils in blood. Some studies have shown that macrophages are involved in the initial phase of lung injury through production of inflammatory mediators or by changes in barrier function $[35,36]$. Possibly, the recruitment of macrophages to the site of inflammation altered the alveolar permeability, which resulted in recruitment of neutrophils to the lungs. The presence of neutrophils in the airspace is a consistent feature of lung injury in animals and humans because these are the first cells of the immune system to be recruited to the site of inflammation [37]. In addition, we observed that MV led to recruitment of lymphocytes to the lung; our results 
TABLE 3: Biomarkers of inflammation on pulmonary parenchyma.

\begin{tabular}{lcccc}
\hline & CG & DG & MVG & DMVG \\
\hline CCL2 $(\mathrm{pg} / \mathrm{mL})$ & $536.6 \pm 95.15$ & $446.7 \pm 108.2$ & $1896 \pm 273.9^{\mathrm{a}, \mathrm{b}}$ & $1465 \pm 237.6^{\mathrm{a}, \mathrm{b}}$ \\
CCL5 $(\mathrm{pg} / \mathrm{mL})$ & $334.4 \pm 56.37$ & $176.4 \pm 30.36$ & $258.8 \pm 69.23$ & $316.5 \pm 39.74$ \\
IL-17 $(\mathrm{pg} / \mathrm{mL})$ & $1065 \pm 33.61$ & $1060 \pm 31.40$ & $790.9 \pm 26.78^{\mathrm{a}, \mathrm{b}}$ & $884.2 \pm 52.74^{\mathrm{a}, \mathrm{b}}$ \\
IL-22 $(\mathrm{pg} / \mathrm{mL})$ & $185.8 \pm 38.4$ & $207.6 \pm 50.18$ & $753 \pm 96.97^{\mathrm{a}, \mathrm{b}}$ & $619.9 \pm 95.65^{\mathrm{a}, \mathrm{b}}$ \\
IL-10 $(\mathrm{pg} / \mathrm{mL})$ & $3543 \pm 150.70$ & $3367 \pm 99.34$ & $2436 \pm 33.12^{\mathrm{a}, \mathrm{b}}$ & $2820 \pm 67.87^{\mathrm{a}, \mathrm{b}}$ \\
\hline
\end{tabular}

(a) represents significant difference between groups when compared to CG. (b) represents significant difference between groups when compared to DG. Data are expressed as mean \pm standard error of the mean $(n=10)$ and were analyzed by one-way ANOVA followed by Tukey's posttest $(p<0.05)$. CG: control group; DG: diet group; MVG: mechanical ventilation group; DMVG: diet mechanical ventilation group; IL-10: interleukin-10; IL-17: interleukin-17; IL-22: interleukin-22.

TABLE 4: Biomarkers of oxidative stress on pulmonary parenchyma.

\begin{tabular}{lcccc}
\hline & CG & DG & MVG & DMVG \\
\hline SOD (U/mg ptn) & $23.33 \pm 1.98$ & $28.83 \pm 3.60$ & $43.33 \pm 7.18^{\mathrm{a}}$ & $37.32 \pm 2.19^{\mathrm{a}}$ \\
CAT (U/mg ptn) & $1.01 \pm 0.06$ & $0.57 \pm 0.07^{\mathrm{a}}$ & $0.19 \pm 0.02^{\mathrm{a}, \mathrm{b}}$ & $0.13 \pm 0.01^{\mathrm{a}, \mathrm{b}}$ \\
GSH/GSSG ratio & $6.38 \pm 1.64$ & $5.47 \pm 0.60$ & $1.97 \pm 0.18^{\mathrm{a}}$ & $5.05 \pm 1.52$ \\
TBARS (nmol/mg ptn) & $2.42 \pm 0.27$ & $2.16 \pm 0.21$ & $5.85 \pm 1.11^{\mathrm{a}, \mathrm{b}, \mathrm{d}}$ & $2.77 \pm 0.32$ \\
Protein carbonyl (nmol/mg ptn) & $4.74 \pm 0.89$ & $3.45 \pm 0.37$ & $14.20 \pm 1.64^{\mathrm{a}, \mathrm{b}}$ & $18.46 \pm 4.34^{\mathrm{a}, \mathrm{b}}$ \\
\hline
\end{tabular}

(a) represents significant difference between groups when compared to CG. (b) represents significant difference between groups when compared to DG. (d) represents significant difference between groups when compared to DMVG. Data are expressed as mean \pm standard error of the mean $(n=10)$ and were analyzed by one-way ANOVA followed by Tukey's posttest $(p<0.05)$. CG: control group; DG: diet group; MVG: mechanical ventilation group; DMVG: diet mechanical ventilation group; CAT: catalase; GSH: glutathione reduced; GSSG: glutathione oxidized; SOD: superoxide dismutase; TBARS: thiobarbituric acid reactive substances.

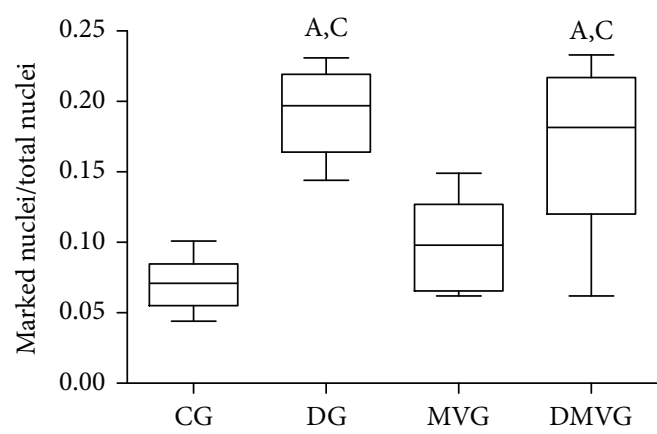

(a)

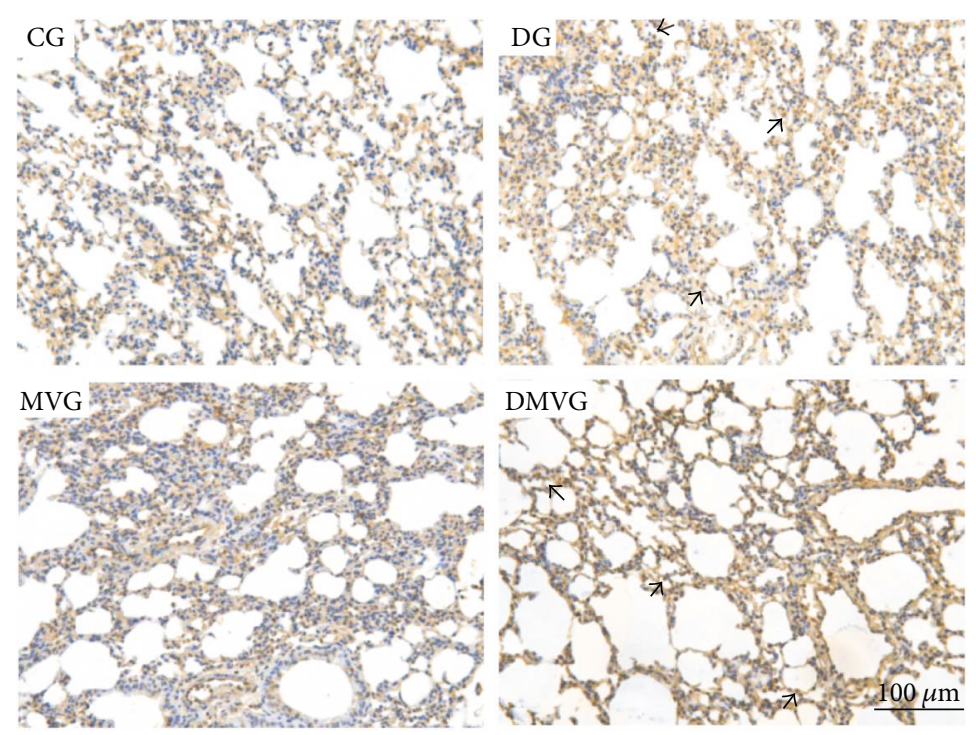

(b)

FIGURE 2: Immunohistochemistry for HMGB1. (a) Ratio between the number of nuclei labeled for HMGB1 antibody and the total number of nuclei. Data are expressed as mean \pm standard error of the mean $(n=10)$. (A) and $(C)$ represent a significant difference in relation to CG and MVG $(p<0.05)$ using Kruskal-Wallis analysis followed by Dunn's posttest. (b) Histological section of lung parenchyma stained by immunohistochemical technique. Bar $=100 \mu \mathrm{m}$. The arrows point to marked nuclei.

corroborate the findings of Chess et al. [38] who reported a higher percentage of lymphocytes in ventilated animals with a moderate tidal volume.
Cytokines and chemokines are produced at the site of inflammation by different cells of the innate and adaptive immune system, such as monocytes and neutrophils [1]. 


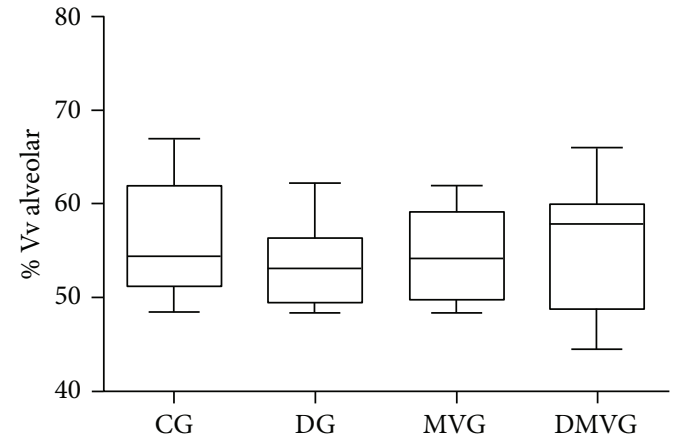

(a)

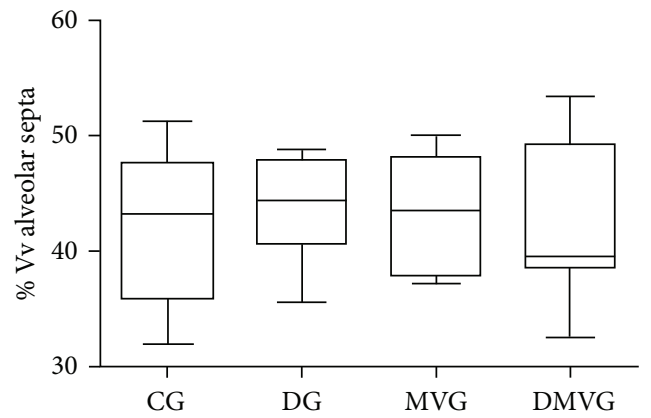

(b)

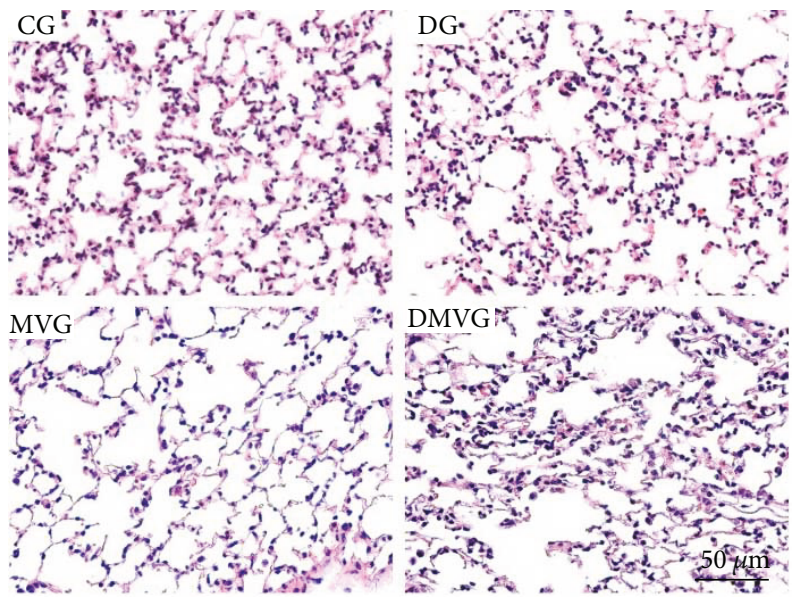

(c)

FIGURE 3: Stereological analyses of lung sections. (a) Volume density of alveolar septa. (b) Volume density of alveolar airspace. (c) Histological section of lung parenchyma stained with $\mathrm{H} \& \mathrm{E}$. Bar $=50 \mu \mathrm{m}$.

Using immunoenzymatic assays performed on lung homogenates, we found decreased IL-17 and increased IL-22 levels in the groups subjected to MV. Interleukin-17 is a proinflammatory interleukin involved in the recruitment of neutrophils to the site of inflammation [39]. Interleukin-22 may have anti- or proinflammatory functions: in the presence of IL-17, IL-22 promotes inflammation in the airways, but in the absence of this IL-17, IL-22 may have a protective role in the airways [40]. Previous studies have shown that IL-22 administration has protective effects in lung injury [41, 42]. In our mechanical ventilation model, interleukin-22 exerts a protective action, as it is increased in groups showing decreased IL-17 levels. IL-22 is related to recruitment of the innate immune cells and increased chemokine production [43]. In our study, the ventilated animals presented with increased CCL2. Ikeuchi et al. [44] observed that IL-22 induced CCL2 expression in rheumatoid arthritis, and studies have shown increased CCL2 levels in animals ventilated with moderate tidal volumes [45]. Our results suggest that CCL2 production was increased for IL-22 to repair the possible tissue damage caused by the antiphysiological mechanism of MV.

In our study, the groups subjected to MV presented a decrease in IL-10 compared to the animals kept on spontaneous ventilation. IL-10 is an anti-inflammatory cytokine that may decrease or inhibit the synthesis or secretion of inflammatory factors [46]. Some studies have observed a reduction in IL-10 and an increase in the production of inflammatory cytokines in an experimental model and clinical trial of mechanical ventilation $[47,48]$. Considering previous findings, we believe that the acute inflammatory process triggered by MV leads to a reduction in interleukin production, which may contribute to the development of lung injury.

The recruitment of inflammatory cells to the pulmonary parenchyma is related to the production of oxidants and may induce acute lung injury [49]. Kavazis et al. [50] demonstrated that mechanical ventilation leads to oxidative stress. We analyzed the oxidative damage, and in the two groups submitted to MV, increased protein oxidation was observed. In addition we analyzed lipid oxidation, and there are two main ways to assess lipid peroxidation: by measuring the formation of alkoxyl radicals, as analyzed in this study, and by measuring the formation of the peroxyl radical [51]. In this study, in the animals of the mechanical ventilation group (MVG), we observed only the effect of mechanical ventilation in the lungs of the mice; however, the animals in the diet mechanical ventilation group (DMVG) previously ventilated received the high-fat diet for 10 weeks. Thus, due to the nutritional protocol to which the animals were submitted to, lipid oxidation may have occurred through the formation 
of isoprostanes. Chacon-Cabrera et al. [52] suggested that MV leads to oxidative damage only when used with a nonphysiological tidal volume. Our results differ from those of previous studies, as we have shown that in animals without prior lung injury, mechanical ventilation leads to oxidative stress. In order to counterbalance the reactive species, the lungs present an antioxidant defense system that includes the SOD, CAT, and GPx enzymes [53]. In our study, we observed an increase in the inflammatory cells in the pulmonary parenchyma in groups submitted to mechanical ventilation, and it is known that macrophages and neutrophils contribute to the increase in the production of reactive oxygen species. In this context, the groups subjected to MV showed an increase in SOD activity. The increase of superoxide dismutase activity can be observed, since SOD is one of the first enzymes of the antioxidant defense system to act in the removal of the reactive species [54]. Concentrations of hydrogen peroxide have increased as a result of higher SOD activity. Hydrogen peroxide removal will occur by the action of both catalase and glutathione peroxidase [54]. In our study, we observed a decrease in CAT activity and GSH/GSSG ratio. Marín-Corral et al. [55] observed that in animals ventilated with a moderate tidal volume, there was a reduction in catalase activity. Previous studies have reported a reduction in catalase activity in the lung and liver of rats fed a high-fat diet $[56,57]$. The result of our study corroborates that of the previous studies; we believe that mechanical ventilation and consumption of a diet rich in saturated lipids altered oxygen metabolism, increasing the production of reactive species, which led to depletion of catalase reserves [58]. Andrade et al. [59] and Pires et al. [60] demonstrated a reduction in GSH/GSSG ratio in rats and mice submitted to mechanical ventilation, using a tidal volume similar to the used in this study. Reddy et al. [61] reported that exposure of epithelial cells to cyclic stretching caused a significant reduction in this ratio.

The changes in pulmonary histoarchitecture are mediated by protein oxidation, peroxidation of membrane lipids, and DNA strand breakage; inflammatory cells such as macrophages and neutrophils are also involved in lung architecture remodeling $[62,63]$. In our study, although the results showed cell recruitment and oxidative damage, we did not find any alterations in pulmonary histoarchitecture; we believe that the short time of mechanical ventilation and the ventilation strategy used might have influenced this result.

\section{Conclusions}

The present study has some limitations; we could not analyze the ventilatory mechanics and hemodynamics of the animals on ventilation. These data would allow the determination of the influence of the high-fat diet and the mechanical ventilation on respiratory physiology. However, for our data set, it is possible to conclude that the mechanical ventilation and its association with obesity promoted inflammation and pulmonary oxidative stress in adult mice.

\section{Conflicts of Interest}

The authors declare that they have no competing interests in this study.

\section{Acknowledgments}

The authors would like to express their gratitude to the Foundation for Research Support of Minas Gerais (FAPEMIG) that supported this work by the "Announcement 01/2016-Universal Demand" (process no. CDS APQ00823-16), and to the Federal University of Ouro Preto (UFOP). André Talvani credits $\mathrm{CNPq}$ for the fellowship of research productivity.

\section{References}

[1] H. Tilg and A. R. Moschen, "Adipocytokines: mediators linking adipose tissue, inflammation and immunity," Nature Reviews Immunology, vol. 6, no. 10, pp. 772-783, 2006.

[2] WHO, Obesity: Preventing and Managing the Global Epidemic: Report of a WHO Consultation, WHO, Geneva, Switzerland, 2000.

[3] R. P. P. De Francischi, L. O. Pereira, C. S. Freitas et al., "Obesity: updated information about its etiology, morbidity and treatment," Revista de Nutrição, vol. 13, no. 1, pp. 17-28, 2000.

[4] N. Ouchi, J. L. Parker, J. J. Lugus, and K. Walsh, "Adipokines in inflammation and metabolic disease," Nature Reviews Immunology, vol. 11, no. 2, pp. 85-97, 2011.

[5] J. C. Sebastian, "Respiratory physiology and pulmonary complications in obesity," Best Practice \& Research Clinical Endocrinology \& Metabolism, vol. 27, no. 2, pp. 157-161, 2013.

[6] S. M. Koenig, "Pulmonary complications of obesity," The American Journal of the Medical Sciences, vol. 321, no. 4, pp. 249-279, 2001.

[7] R. L. Jones and M.-M. U. Nzekwu, "The effects of body mass index on lung volumes," Chest, vol. 130, no. 3, pp. 827-833, 2006.

[8] S. Tafelski, H. Yi, F. Ismaeel, A. Krannich, C. Spies, and I. Nachtigall, "Obesity in critically ill patients is associated with increased need of mechanical ventilation but not with mortality," Journal of Infection and Public Health, vol. 9, no. 5, pp. 577-585, 2016.

[9] V. Lionetti, F. A. Recchia, and V. Marco Ranieri, "Overview of ventilator-induced lung injury mechanisms," Current Opinion in Critical Care, vol. 11, no. 1, pp. 82-86, 2005.

[10] O. Syrkina, B. Jafari, C. A. Hales, and D. A. Quinn, "Oxidant stress mediates inflammation and apoptosis in ventilatorinduced lung injury," Respirology, vol. 13, no. 3, pp. 333-340, 2008.

[11] F. Holguin, "Oxidative stress in airway diseases," Annals of the American Thoracic Society, vol. 10, pp. S150-S157, 2013.

[12] V. I. Lushchak, "Free radicals, reactive oxygen species, oxidative stress and its classification," Chemico-Biological Interactions, vol. 224, pp. 164-175, 2014.

[13] S. R. de Noronha, G. V. Campos, A. R. Abreu, A. A. de Souza, D. A. Chianca Jr, and R. C. de Menezes, "High fat diet inducedobesity facilitates anxiety-like behaviors due to GABAergic impairment within the dorsomedial hypothalamus in rats," Behavioural Brain Research, vol. 316, pp. 38-46, 2017. 
[14] K. B. Pena, C. O. Ramos, N. P. Soares et al., "The administration of a high refined carbohydrate diet promoted an increase in pulmonary inflammation and oxidative stress in mice exposed to cigarette smoke," International Journal of Chronic Obstructive Pulmonary Disease, vol. 11, pp. 3207-3217, 2016.

[15] K. K. D. Campos, G. R. Araújo, T. L. Martins et al., “The antioxidant and anti-inflammatory properties of lycopene in mice lungs exposed to cigarette smoke," The Journal of Nutritional Biochemistry, vol. 48, pp. 9-20, 2017.

[16] M. Catta-Preta, M. A. Martins, T. M. Cunha Brunini, A. C. Mendes-Ribeiro, C. A. Mandarim-de-Lacerda, and M. B. Aguila, "Modulation of cytokines, resistin, and distribution of adipose tissue in $\mathrm{C}_{57} \mathrm{BL} / 6$ mice by different high-fat diets," Nutrition, vol. 28, no. 2, pp. 212-219, 2012.

[17] C. A. Mandarim-de-Lacerda, "Stereological tools in biomedical research," Anais da Academia Brasileira de Ciências, vol. 75, no. 4, pp. 469-486, 2003.

[18] N. P. Soares, K. K. D. Campos, K. B. Pena et al., "The effects of the combination of a refined carbohydrate diet and exposure to hyperoxia in mice," Oxidative Medicine and Cellular Longevity, vol. 2016, Article ID 1014928, 11 pages, 2016.

[19] M. F. Ricci, C. F. Campos, C. T. Cartelle et al., "Nitrergic myenteric neurons are spared in experimental chagasic megacolon," Journal of Neuroinfectious Diseases, vol. 7, no. 4, p. 235, 2016.

[20] S. Marklund and G. Marklund, "Involvement of the superoxide anion radical in the autoxidation of pyrogallol and a convenient assay for superoxide dismutase," European Journal of Biochemistry, vol. 47, no. 3, pp. 469-474, 1974.

[21] H. Aebi, "[13] Catalase in vitro," Methods in Enzymology, vol. 105, no. 1947, pp. 121-126, 1984.

[22] O. W. Griffith, "Determination of glutathione and glutathione disulfide using glutathione reductase and 2-vinylpyridine," Analytical Biochemistry, vol. 106, no. 1, pp. 207-212, 1980.

[23] J. A. Buege and S. D. Aust, "[30] Microsomal lipid peroxidation," Methods in Enzymology, vol. 52, pp. 302-310, 1978.

[24] A. Z. Reznick and L. Packer, "[38] Oxidative damage to proteins: spectrophotometric method for carbonyl assay," Methods in Enzymology, vol. 233, no. 1991, pp. 357-363, 1994.

[25] M. M. Bradford, "A rapid and sensitive method for the quantitation of microgram quantities of protein utilizing the principle of protein-dye binding," Analytical Biochemistry, vol. 72, no. 1-2, pp. 248-254, 1976.

[26] R. T. Enos, J. M. Davis, K. T. Velázquez et al., "Influence of dietary saturated fat content on adiposity, macrophage behavior, inflammation, and metabolism: composition matters," Journal of Lipid Research, vol. 54, no. 1, pp. 152-163, 2013.

[27] P. A. Kakimoto and A. J. Kowaltowski, "Effects of high fat diets on rodent liver bioenergetics and oxidative imbalance," Redox Biology, vol. 8, pp. 216-225, 2016.

[28] R. A. van der Heijden, F. Sheedfar, M. C. Morrison et al., "High-fat diet induced obesity primes inflammation in adipose tissue prior to liver in C57BL/6j mice," Aging, vol. 7, no. 4, pp. 256-268, 2015.

[29] A. V. M. Ferreira, Z. Menezes-Garcia, J. B. Viana, É. G. Mário, and L. M. Botion, "Distinct metabolic pathways trigger adipocyte fat accumulation induced by high-carbohydrate and highfat diets," Nutrition, vol. 30, no. 10, pp. 1138-1143, 2014.

[30] M. K. Gunasekaran, W. Viranaicken, A. C. Girard et al., "Inflammation triggers high mobility group box 1 (HMGB1) secretion in adipose tissue, a potential link to obesity," Cytokine, vol. 64, no. 1, pp. 103-111, 2013.
[31] V. N. Montes, S. Subramanian, L. Goodspeed et al., "AntiHMGB1 antibody reduces weight gain in mice fed a high-fat diet," Nutrition \& Diabetes, vol. 5, no. 6, article e161, 2015.

[32] C. Yildiz, N. Palaniyar, G. Otulakowski et al., "Mechanical ventilation induces neutrophil extracellular trap formation," Anesthesiology, vol. 114, no. 4, pp. 864-875, 2015.

[33] J. C. McNelis and J. M. Olefsky, "Macrophages, immunity, and metabolic disease," Immunity, vol. 41, no. 1, pp. 36-48, 2014.

[34] H. Tashiro, K. Takahashi, H. Sadamatsu et al., "Saturated fatty acid increases lung macrophages and augments house dust mite-induced airway inflammation in mice fed with high-fat diet," Inflammation, vol. 40, no. 3, pp. 1072-1086, 2017.

[35] J. A. Frank, C. M. Wray, D. F. McAuley, R. Schwendener, and M. A. Matthay, "Alveolar macrophages contribute to alveolar barrier dysfunction in ventilator-induced lung injury," American Journal of Physiology-Lung Cellular and Molecular Physiology, vol. 291, no. 6, pp. L1191-L1198, 2006.

[36] F. G. Eyal, C. R. Hamm, and J. C. Parker, "Reduction in alveolar macrophages attenuates acute ventilator induced lung injury in rats," Intensive Care Medicine, vol. 33, no. 7, pp. 1212-1218, 2007.

[37] J. Grommes and O. Soehnlein, "Contribution of neutrophils to acute lung injury," Molecular Medicine, vol. 17, no. 3-4, pp. 1307, 2011.

[38] P. R. Chess, R. P. Benson, W. M. Maniscalco, T. W. Wright, M. A. O'Reilly, and C. J. Johnston, "Murine mechanical ventilation stimulates alveolar epithelial cell proliferation," Experimental Lung Research, vol. 36, no. 6, pp. 331-341, 2010.

[39] K. Eyerich, V. Dimartino, and A. Cavani, "IL-17 and IL-22 in immunity: driving protection and pathology," European Journal of Immunology, vol. 47, no. 4, pp. 607-614, 2017.

[40] S. Rutz, C. Eidenschenk, and W. Ouyang, "IL-22, not simply a Th17 cytokine," Immunological Reviews, vol. 252, no. 1, pp. 116-132, 2013.

[41] Z. Wu, Z. Hu, X. Cai et al., "Interleukin 22 attenuated angiotensin II induced acute lung injury through inhibiting the apoptosis of pulmonary microvascular endothelial cells," Scientific Reports, vol. 7, no. 1, p. 2210, 2017.

[42] S. Hoegl, M. Bachmann, P. Scheiermann et al., "Protective properties of inhaled IL-22 in a model of ventilator-induced lung injury," American Journal of Respiratory Cell and Molecular Biology, vol. 44, no. 3, pp. 369-376, 2011.

[43] S. J. Aujla, Y. R. Chan, M. Zheng et al., "IL-22 mediates mucosal host defense against gram-negative bacterial pneumonia," Nature Medicine, vol. 14, no. 3, pp. 275-281, 2008.

[44] H. Ikeuchi, T. Kuroiwa, N. Hiramatsu et al., "Expression of interleukin-22 in rheumatoid arthritis: potential role as a proinflammatory cytokine," Arthritis \& Rheumatism, vol. 52, no. 4, pp. 1037-1046, 2005.

[45] W. A. Altemeier, G. Matute-Bello, C. W. Frevert et al., "Mechanical ventilation with moderate tidal volumes synergistically increases lung cytokine response to systemic endotoxin," American Journal of Physiology-Lung Cellular and Molecular Physiology, vol. 287, no. 3, pp. L533-L542, 2004.

[46] R. L. Hawwa, M. A. Hokenson, Y. Wang, Z. Huang, S. Sharma, and J. Sanchez-Esteban, "IL-10 inhibits inflammatory cytokines released by fetal mouse lung fibroblasts exposed to mechanical stretch," Pediatric Pulmonology, vol. 46, no. 7, pp. 640-649, 2011. 
[47] B. Bohrer, R. C. Silveira, E. C. Neto, and R. S. Procianoy, "Mechanical ventilation of newborns infant changes in plasma pro- and anti-inflammatory cytokines," The Journal of Pediatrics, vol. 156, no. 1, pp. 16-19, 2010.

[48] H.-S. Lee and C.-K. Kim, "Effect of recombinant IL-10 on cultured fetal rat alveolar type II cells exposed to 65\%-hyperoxia," Respiratory Research, vol. 12, no. 1, p. 68, 2011.

[49] M. A. Matthay and R. L. Zemans, "The acute respiratory distress syndrome: pathogenesis and treatment," Annual Review of Pathology, vol. 6, no. 1, pp. 147-163, 2011.

[50] A. N. Kavazis, E. E. Talbert, A. J. Smuder, M. B. Hudson, W. B. Nelson, and S. K. Powers, "Mechanical ventilation induces diaphragmatic mitochondrial dysfunction and increased oxidant production," Free Radical Biology \& Medicine, vol. 46, no. 6, pp. 842-850, 2009.

[51] C. R. Rueff-barroso, E. T. L. Trajano, J. N. Alves et al., "Organ-related cigarette smoke-induced oxidative stress is strain-dependent," Medical Science Monitor, vol. 16, no. 7, pp. 218-226, 2010.

[52] A. Chacon-Cabrera, Y. Rojas, L. Martínez-Caro et al., "Influence of mechanical ventilation and sepsis on redox balance in diaphragm, myocardium, limb muscles, and lungs," Translational Research, vol. 164, no. 6, pp. 477-495, 2014.

[53] L. A. Pham-Huy, H. He, and C. Pham-Huy, "Free radicals, antioxidants in disease and health," International Journal of Biomedical Sciences, vol. 4, no. 2, pp. 89-96, 2008.

[54] E. Birben, U. M. Sahiner, C. Sackesen, S. Erzurum, and O. Kalayci, "Oxidative stress and antioxidant defense," World Allergy Organization Journal, vol. 5, no. 1, pp. 9-19, 2012.

[55] J. Marín-Corral, L. Martínez-Caro, J. A. Lorente et al., "Redox balance and cellular inflammation in the diaphragm, limb muscles, and lungs of mechanically ventilated rats," Anesthesiology, vol. 112, no. 2, pp. 384-394, 2010.

[56] L. Ashakumary and P. L. Vijayammal, "Effect of nicotine on antioxidant defence mechanisms in rats fed a high-fat diet," Pharmacology, vol. 52, no. 3, pp. 153-158, 1996.

[57] R. Vuković, S. Blažetić, I. Oršolić et al., "Impact of ovariectomy, high fat diet, and lifestyle modifications on oxidative/ antioxidative status in the rat liver," Croatian Medical Journal, vol. 55, no. 3, pp. 218-227, 2014.

[58] A. Fernández-Sánchez, E. Madrigal-Santillán, M. Bautista et al., "Inflammation, oxidative stress, and obesity," International Journal of Molecular Sciences, vol. 12, no. 12, pp. 3117-3132, 2011.

[59] M. C. Andrade, S. ABF, J. G. Horta et al., "Applying positive end-expiratory pressure during mechanical ventilation causes pulmonary redox imbalance and inflammation in rats," Shock, p. 1, 2017.

[60] K. M. Pires, A. C. Melo, M. Lanzetti et al., "Low tidal volume mechanical ventilation and oxidative stress in healthy mouse lungs," Jornal Brasileiro de Pneumologia, vol. 38, no. 1, pp. 98-104, 2012.

[61] S. P. Reddy, P. M. Hassoun, and R. Brower, "Redox imbalance and ventilator-induced lung injury," Antioxidants \& Redox Signaling, vol. 9, no. 11, pp. 2003-2012, 2007.
[62] K. K. D. Campos, S. F. Leal, D. C. Costa, W. G. de Lima, and F. S. Bezerra, "Long-term exposure to ultrasonically nebulized distilled water and saline causes cellular influx and oxidative stress in lung tissue of rats," Experimental Lung Research, vol. 41, no. 10, pp. 546-553, 2015.

[63] S. S. Valença and L. C. Porto, "Estudo imunohistoquímico do remodelamento pulmonar em camundongos expostos à fumaça de cigarro," Jornal Brasileiro de Pneumologia, vol. 34, no. 10 , pp. $787-795,2008$. 


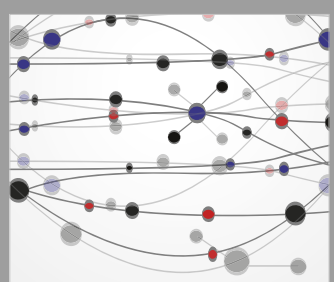

The Scientific World Journal
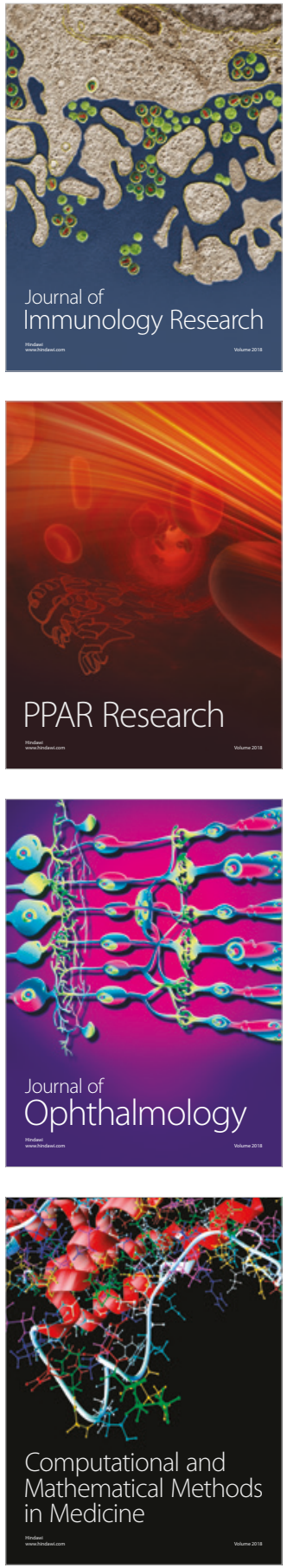

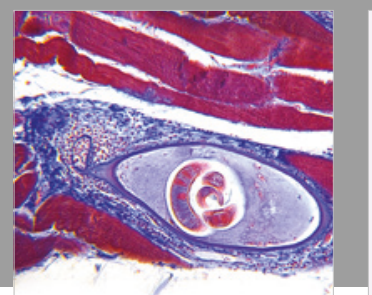

Gastroenterology Research and Practice

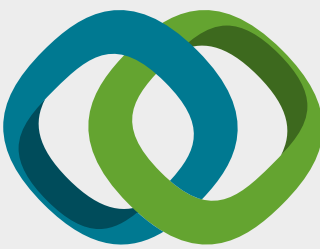

\section{Hindawi}

Submit your manuscripts at

www.hindawi.com
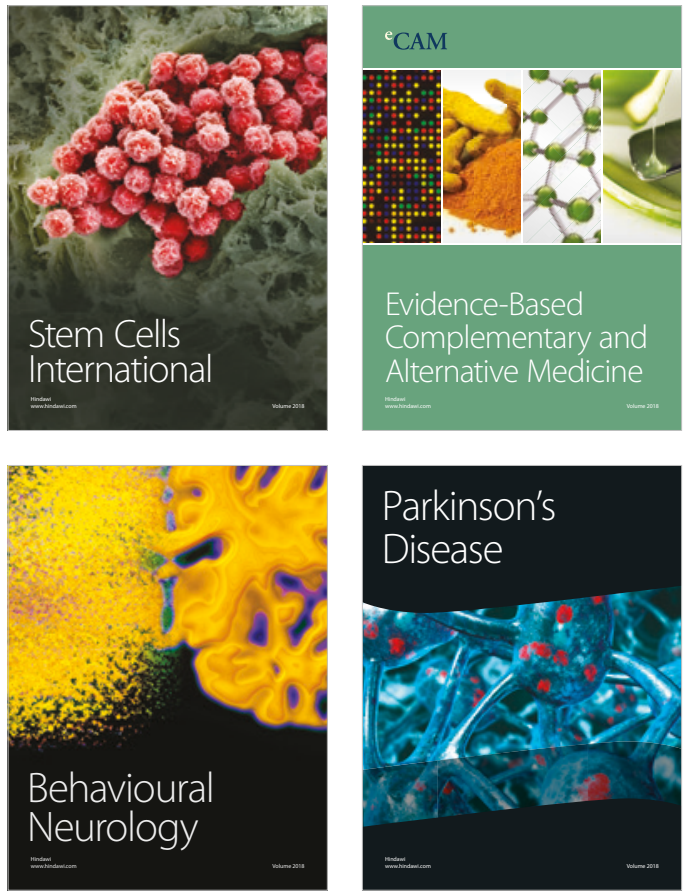

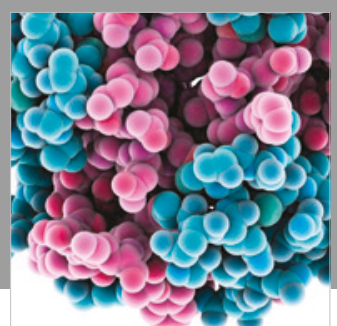

ournal of

Diabetes Research

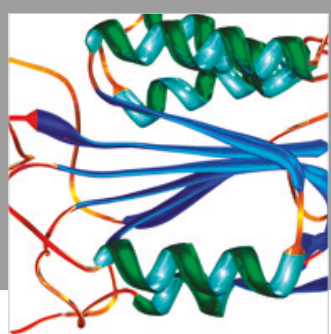

Disease Markers
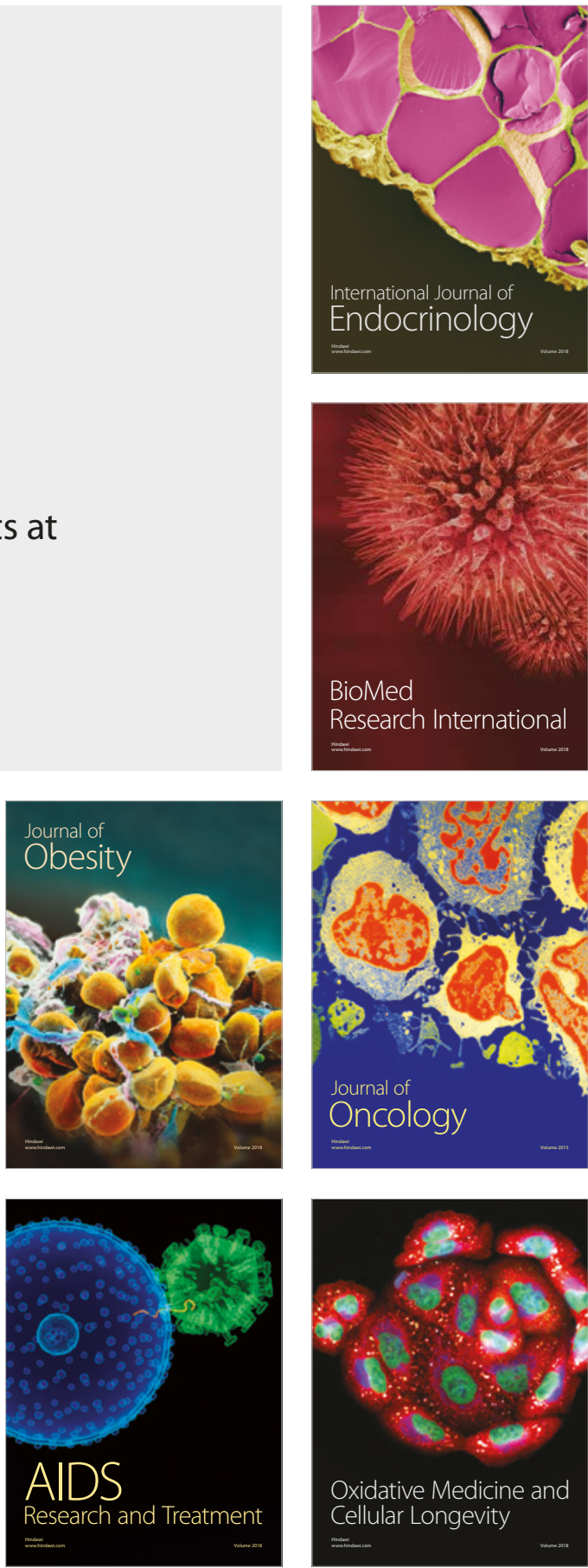